\title{
Neuronal Correlates of Memory Formation in Motor Cortex after Adaptation to Force Field
}

\author{
Fritzie Arce, ${ }^{1,4}$ Itai Novick, ${ }^{1,4}$ Yael Mandelblat-Cerf, ${ }^{1,4}$ and Eilon Vaadia ${ }^{1,2,3,4}$ \\ ${ }^{1}$ Department of Medical Neurobiology, The Institute for Medical Research Israel-Canada, Hadassah Medical School, ${ }^{2}$ The Interdisciplinary Center for \\ Neural Computation, ${ }^{3}$ Edmond and Lily Safra Center for Brain Sciences, and ${ }^{4}$ Hebrew University, Jerusalem 91120, Israel
}

Activity of single neurons in the motor cortex has been shown to change during acquisition of motor skills. We previously reported that the combined activity of cell ensembles in the motor cortex of monkeys (Macaca fascicularis) evolves during adaptation to a novel force field perturbation to encode the direction of compensatory force when reaching to visual targets. We also showed that the population directional signal was altered by the available sensory feedback. Here, we examined whether traces of such activity would linger on to later constitute motor memories of the newly acquired skill and whether memory traces would differ depending on feedback. We found that motor-cortical cell ensembles retained features of their adaptive activity pattern in the absence of perturbation when reaching to both learned and unlearned targets. Moreover, the preferred directions of these cells rotated in the direction of force field while the entire population of cells produced no net rotation of preferred direction when returning to null-field reaches. Whereas the activity pattern and preferred direction rotations were comparable with and without visual feedback, changes in tuning amplitudes differed across feedback conditions. Last, savings in behavioral performance and neuronal activity during later reexposure to force field were apparent. Overall, the findings reflect a novel representation of motor memory by cell ensembles and indicate a putative role of the motor cortex in early acquisition of motor memory.

\section{Introduction}

Acquisition of motor skills involves a process of initial encoding of new motor outputs in response to novel sensorimotor associations (Wise et al., 1998; Gandolfo et al., 2000; Li et al., 2001; Paz et al., 2003; Lebedev et al., 2005; Jarosiewicz et al., 2008; Zach et al., 2008; Arce et al., 2010). Unless disrupted, this may be followed by a process of consolidation in which labile motor memories may evolve into a more stable form, becoming less susceptible to interference and allowing for later retrieval (Stickgold and Walker, 2005; Nader and Hardt, 2009; Robertson, 2009). Indeed, performance "savings" (i.e., a faster rate of improvement on retest on a learned task compared with the initial exposure) indicates stabilization of recently acquired memories. Motor memories may also be enhanced on retrieval and subsequently stored in a modified form (Misanin et al., 1968; Nader, 2003).

Several studies at the cellular and systems level have explored the neural substrates underlying memory encoding, storage, and retrieval (Thompson, 2005; Silva et al., 2009). Multiple brain

Received March 29, 2010; revised May 20, 2010; accepted May 26, 2010.

This work was supported in part by the United States-Israel Binational Science Foundation, by the Israeli Science Foundation, and by special contributions by The Rosetrees Trust and The Ida Baruch Fund. E.V. is the Jack H. Skirball Chair of Brain Research. We thank Claude Ghez for recommendations on the experimental design, Hagai Lalazar for insightful discussions, Sharon Freeman for assistance in the recordings, and Dmitry Davidov and Ben Engelhard for programming and technical assistance.F.A. and E.V. designed the experiment; F.A., I.N., Y.M.-C., and E.V. performed the experiments; F.A. analyzed the data; and F.A. and E.V. wrote the paper.

The authors declare no competing financial interests.

Correspondence should be addressed to Dr. Fritzie Arce at her present address: Department of Organismal Biology and Anatomy, University of Chicago, 1027 East 57th Street, Chicago, IL 60637. E-mail: fritziea@uchicago.edu.

DOI:10.1523/JNEUROSCI.1603-10.2010

Copyright $\odot 2010$ the authors $\quad 0270-6474 / 10 / 309189-10 \$ 15.00 / 0$ areas (e.g., hippocampus, amygdala, cerebral cortex, striatum, cerebellum) seem to be involved in these processes (Attwell et al., 2002; Ribeiro and Nicolelis, 2004; Kassardjian et al., 2005; Ji and Wilson, 2007; Montgomery and Buzsáki, 2007; Karlsson and Frank, 2009). For example, similar cell ensembles in the amygdala of transgenic mice were found to be active during learning and retrieval of fear memory (Reijmers et al., 2007). A recent study showed erasure of fear memory when a specific subpopulation of neurons in the amygdala was ablated (Han et al., 2009).

Human behavioral studies using functional imaging and transcranial magnetic stimulation indicate involvement of the motor cortex in memory acquisition and consolidation (Karni et al., 1995; Muellbacher et al., 2002; Baraduc et al., 2004; Richardson et al., 2006). Electrophysiology studies have shown reactivation of recent memory traces in the motor areas of the zebra finch brain (Dave and Margoliash, 2000) and primate motor cortex during rest (Hoffman and McNaughton, 2002). However, the scant existing electrophysiological evidence cannot conclusively determine whether the same motor cortical neurons activated during learning are also reactivated outside of the learning episode or whether memory retrieval is disrupted if the activation of these neurons is inhibited. This would indicate whether similar neural circuits underlie learning and memory. Furthermore, it remains unclear whether and how the context of learning, such as available sensory feedback, influences the selection of neurons that represent the memory trace.

We previously reported that specific motor-cortical cell ensembles were preferentially recruited during adaptation to force fields, as their firing rates were modulated depending on the directional tuning properties of the cells (Arce et al., 2010). There, 
we suggested that the summed activity of these cell ensembles constitute the basis function with which the motor output is computed. Here, we assessed the putative role of the motor cortex in the generation of early motor memories of the newly learned task and whether the representation of memories would differ depending on the adaptive strategies used based on available feedback. Thus, we compared the directional tuning properties of motor-cortical neurons before and after learning while monkeys made null-field center-out reaches to visual targets.

\section{Materials and Methods}

Animal care and experimentation complied with the National Institutes of Health Guide for the Care and Use of Laboratory Animals and with the Hebrew University guidelines for the use and care of laboratory animals in research, approved and supervised by the Institutional Animal Care and Use Committee.

\section{Behavioral task and recordings}

Details of the experimental procedures were described previously (Arce et al., 2010). Briefly, two monkeys (Macaca fascicularis; $\sim 4 \mathrm{~kg}$; one male and one female) were trained to make center-out reaches to visual targets using a robotic arm (Phantom Premium 1.5 High Force; SensAble Devices). On completion of training, we proceeded with surgery and recording. Monkey A was implanted with a recording chamber $(27 \times 27$ $\mathrm{mm}$ ) above the left hemisphere. Signals from 32 moveable microelectrodes were amplified, filtered, on-line sorted, and sampled at $20 \mathrm{kHz}$ (Alpha-Omega). Monkey B was chronically implanted with a Utah 96microelectrode array (length of $1.5 \mathrm{~mm}$; Blackrock Microsystems) on the arm region of the left primary motor cortex (M1). Signals were on-line sorted and sampled at $30 \mathrm{kHz}$ using a Cerebus acquisition system (Blackrock Microsystems). Recording sites for monkey B corresponded approximately to layer 5 . For monkey A, most recording sites were chosen based on the quality of the isolation of single units. Since large cells with larger spike amplitudes were found in deeper layers, our sample was probably biased around layer 5 . However, given the restrictions of multiday/multichannel recordings, we made no additional efforts to locate the cortical layer of the recording sites.

\section{Task design and apparatus}

We used a local learning paradigm in which a viscous curl force field (FF) was applied during reaches to only one target direction designated as the learned direction (LD). As monkeys reached to the $\mathrm{LD}$, the force field perturbed the hand perpendicular to the direction of reaching and proportional to the movement speed. Monkeys were exposed to the force field only in the recording sessions. To ensure that monkeys learned anew, the learned direction (one of the targets at $0,45,90,135,180^{\circ}$ ) and force field direction (either clockwise or counterclockwise) were varied. The daily sessions consisted of five trial blocks: (1) the prelearning block consisted of null-field reaches to eight different targets (160 trials), (2) the adaptation block consisted of force field reaches to one and the same target (144 FF trials and 16 interspersed catch trials in the identical direction), (3) the postlearning block, with another 160 center-out reaches with a null field, (4) the retest block as in the adaptation block but without catch trials, and (5) the post-retest block as in the postlearning block (see Fig. 1A). In most sessions, monkeys completed only the first three blocks. There were seven sessions with the retest and post-retest blocks. Each recording session was either with or without visual feedback (VFB) of the cursor, which instantaneously tracked the hand position.

\section{Trial flow}

Trials started with the appearance of an origin and a cursor (both $10 \mathrm{~mm}$ radius). The monkey had to position the cursor at the origin and to hold for a randomized variable period $(0.75-1.25 \mathrm{~s})$ until a target appeared (10 $\mathrm{mm}$ radius and $4.24 \mathrm{~cm}$ from the origin). After another randomized variable hold period from target onset $(0.75-1.25 \mathrm{~s})$, the origin disappeared to signal the monkeys to start moving. Monkeys were given $1 \mathrm{~s}$ from the go signal to reach the target. With VFB, the cursor was visible during the reach movement, whereas, without VFB, the cursor disap- peared at target onset and appeared only at trial end. Trial end was defined either by a successful target reach or a failure event (i.e., inability to comply with required hold and movement times). On reaching the target, the monkey had to hold the position for $0.2 \mathrm{~s}$ to get a reward. An intertrial interval $(1.5 \mathrm{~s})$ followed trial end during which the workspace was blanked. In field trials, the velocity-dependent force field (Arce et al., 2010) was generated with $k=8 \mathrm{~N}^{*} \mathrm{~s} / \mathrm{mm}$ and turned off $200 \mathrm{~ms}$ after trial end.

\section{Data analysis}

\section{Behavioral analysis}

Behavioral parameters (e.g., success rate, reaction time, movement duration, and velocity profiles) were compared between prelearning and postlearning blocks. We also evaluated performance using the initial directional deviation (i.e., the angular difference between the directions of two vectors going from the hand position at movement onset to the target and from the origin to the hand position $150 \mathrm{~ms}$ after movement onset).

\section{Neuronal analysis}

Dataset. Criteria for inclusion of cells were (1) stable cell isolation, (2) with average firing rate of $>1 \mathrm{~Hz}$, and (3) at least five successful trials per direction in the prelearning and postlearning blocks separately. Cell activity during the prelearning and postlearning blocks was evaluated for two epochs: (1) the preparatory epoch taken from the hold period $150 \mathrm{~ms}$ from target onset to $600 \mathrm{~ms}$ after and (2) the movement-related epoch from the go signal to $600 \mathrm{~ms}$ after.

Measuring prelearning to postlearning changes in firing rates. Comparisons of mean firing rates corresponding to prelearning and postlearning reaches to direction $d(i)$ (where $i=1-8$ ) were tested for significance (Mann-Whitney, $p<0.05$ ). For cell $k$ that showed significant differences, we calculated a postlearning modulation index as follows:

Modulation index $=\left(\right.$ Spost $\left._{d(i), k}-\operatorname{Spre}_{d(i), k}\right) /\left(\operatorname{Spost}_{d(i), k}+\operatorname{Spre}_{d(i), k}\right)$,

where Spre $_{d(i), k}$ and Spost $d(i), k$ are the mean discharge rates of neuron $k$ during null-field reaches to direction $d(i)$ in the prelearning and postlearning blocks, respectively. The index ranges between -1 and 1 , where positive values reflect increased firing in the postlearning trials.

Measuring dependence of modulation index on the directional tuning of the cells. We tested whether the postlearning modulation indexes depended on the preferred direction (PD) of the cells as found previously when comparing neuronal activity between the prelearning and learning blocks (Arce et al., 2010). To do this, we divided the population of directionally tuned cells into FF-modulated (i.e., cells that showed modulation during the adaptation phase) and non-FF-modulated cells. As done previously (Arce et al., 2010), we first computed the normalized PD (nPD) of each cell as the angular distance between its PD and the learned target direction (LD) and was signed $(+/-)$ depending on the force field direction. Positive denotes nPDs in the direction of the force field, whereas negative denotes nPDs in the opposing direction. We pooled the data across force field directions by flipping the nPD for the counterclockwise force field. We then subdivided the population of cells according to their nPDs: (1) near-LD (LD) $\left(-45\right.$ to $\left.45^{\circ}\right)$, (2) counterfield (CF) $\left(-135\right.$ to $\left.-46^{\circ}\right)$, (3) with-field (WF) $\left(46\right.$ to $\left.135^{\circ}\right)$, and (4) far-LD $(-136$ to $-180^{\circ}$ and 136 to 180$)$. For each of the eight target directions, we obtained the population response by averaging the modulation indexes across cells within the same nPD range. Then, we fitted the population response as a function of the $\mathrm{nPD}$ to a cosine model as follows:

$$
\mathrm{PI}_{r_{i}} \approx a+b^{*} \cos \left(\theta_{r_{i}}-\mathrm{PD}_{\mathrm{pop}}\right)
$$

where $\mathrm{PI}_{r_{i}}$ is the population modulation index per nPD range $r_{i}$ (where $i=1-5$ ), and $\theta_{r_{i}}$ are the angles corresponding to the $\mathrm{nPD}$ range. $\mathrm{PD}_{\text {pop }}$ is the population preferred direction, which indicates the nPD of maximal modulation index, $a$ is the offset, and $b$ is the amplitude. Goodness of fit to cosine was evaluated using stepwise regression of the unbinned data 


\section{A Block flow}
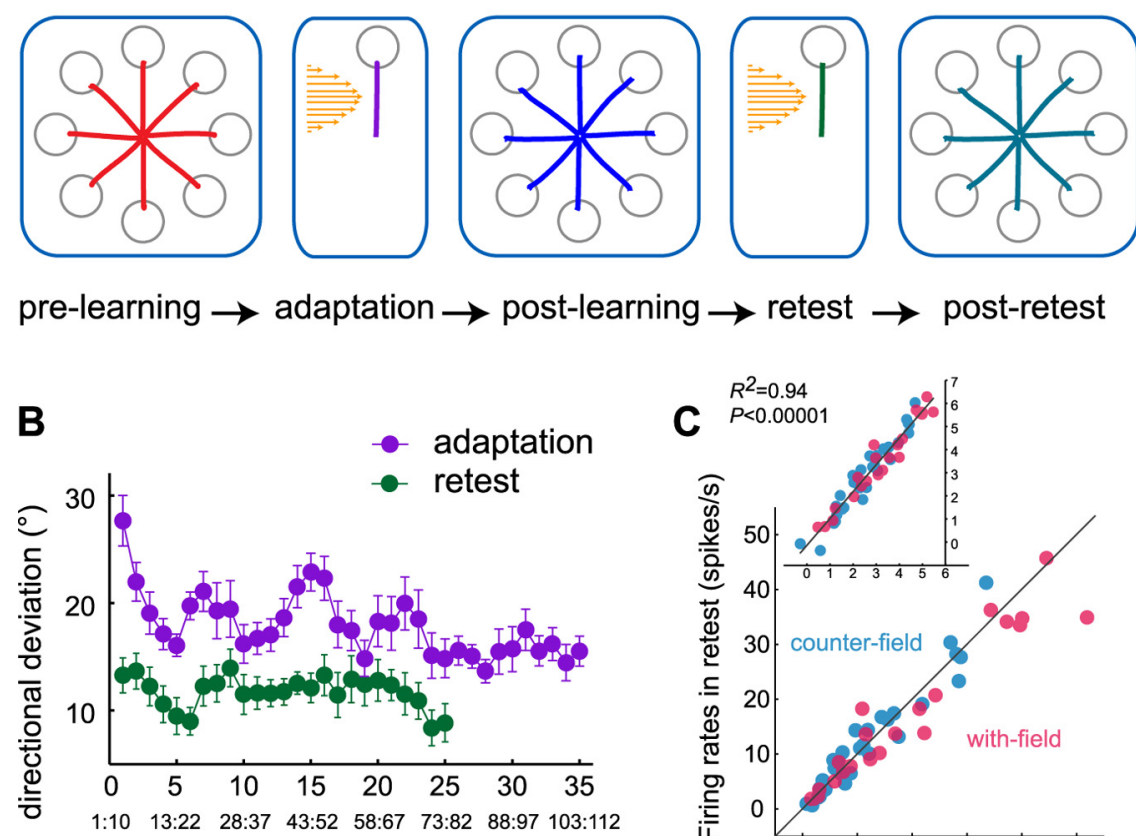

trial bins (10 trials/bin)

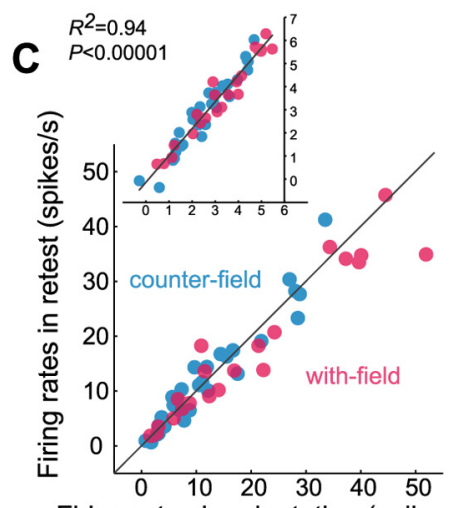

Firing rates in adaptation (spikes/s)

Figure 1. Retest after force field learning. $A$, Daily sessions consisted of five trial blocks: prelearning, adaptation, postlearning, retest, and post-retest. $\boldsymbol{B}$, Mean initial directional deviations during force field adaptation (violet) and retest (green) across sessions with retest block. The vertical bars denote $\pm 1 \mathrm{SE}$. Each point corresponds to mean values across 10 trials. Binning was done by moving three trials forward (see smaller numbers below the trial bin number). C, Correlation between the firing rates during adaptation and retest, averaged across the first 20 trials. Shown for cells with $R^{2}>0.70$. Color coding according to the nPD of the cells that were similar (with-field) or opposite (counterfield) to the direction of force field. The diagonal line marks the unity. The inset shows Box-Cox transformed data in $C$ and the regression fit.

with the first to fifth harmonics as regressors (for details, see Arce et al., 2010). Significance was set at $p<0.05$ for $F$ test with the first harmonic.

Measuring prelearning to postlearning changes in PD and tuning width. For each cell, we constructed separate directional tuning curves for prelearning and postlearning blocks using the target directions. The database used in these analyses included cells that were directionally tuned in both the prelearning and postlearning blocks (i.e., with a significant effect for target direction using a one-way ANOVA and with $\left.R^{2}>0.55\right)$. At the single-unit level, we used circular permutation (Stark and Abeles, 2005) to test for significant shifts in PDs or changes in tuning widths from prelearning to postlearning. To evaluate changes in tuning widths, we calculated an amplitude change index (as described for the modulation index). Population analyses were tested for significance using a onesample $t$ test or sign test depending on sample size and normality of distribution.

Retest analyses. Retest analyses were conducted only for those directionally tuned cells that were recorded stably from the prelearning to the retest block $(N=91$ cells $)$. We used linear regression to evaluate how well the firing rates of the population of cells during adaptation predicted the firing rates at retest. Because the distribution was skewed, we also evaluated the data after a Box-Cox power transformation. We assessed differences between single-cell firing rates during adaptation versus retest using a one-way ANOVA.

All analyses were done using the built-in and custom functions of MATLAB. Significance levels for all tests were set at $p<0.05$.

\section{Results}

We recently reported that specific motor-cortical cell ensembles consistently enhanced or reduced their firing rates depending on their contribution to a direction that compensates for the force perturbation (Arce et al., 2010). These cells are referred to as "FF-modulated cells." Here, we examine whether traces of the newly acquired activity are maintained after learning, or instead are readily extinguished on cessation of the perturbation. To this end, we examined motor cortical activity during performance of the centerout task after exposure to the force field perturbation ("postlearning") and on retest on the learned force field.

\section{Behavior and neuronal activity during retest on force field}

Behavioral savings

Performance savings on retest have often been used to assess the stabilization of motor memories in humans. As expected, we found savings in behavioral performance at retest on the newly learned task after completion of a postlearning block; namely, the initial directional deviations were significantly lower on the first 40 trials of retest compared with those incurred in the adaptation block (Fig. $1 B$ ) (ANOVA, $p<0.0001$ ). This suggests a form of stabilization of the recently acquired motor memory that allowed retrieval.

\section{Neuronal activity during adaptation versus retest}

We have shown recently (Arce et al., 2010) that, during force field adaptation, the activation of FF-modulated cells depended on the angular distance between the PD of the cells and the learned direction. The PD-dependent adaptive modulation followed a cosine model, with increases corresponding to $\mathrm{nPDs}$ opposite the direction of the force field [counterfield $(\mathrm{CF})]$ and decreases corresponding to nPDs similar to the force field direction [with-field (WF)]. We refer to the PDdependent rate modulation as the "adaptive pattern" [Fig. 3, boxed central plots of $A$ (orange) and $B$ (green)]. Here, we found that the firing rates of the cells at retest were well predicted by their firing rates during adaptation (Fig. $1 C)\left(R^{2}=0.94 ; p<0.0001\right)$. Of the 91 cells recorded at retest, 36 were FF-modulated cells that showed similar patterns of rate modulation during adaptation and retest, namely, increases in counterfield cells and decreases in with-field cells. This indicates that these cells have maintained through retest the specific activity pattern acquired during the initial exposure to the force field.

Behavior and neuronal activity during the postlearning block The postlearning block immediately followed the adaptation block in which monkeys reached to eight targets in the absence of force field. Analysis of the first five trials of the postlearning block showed clear behavioral aftereffects; the trajectory deviations were significant in the learned direction (supplemental Fig. $1 C, D$, available at www.jneurosci.org as supplemental material) ( $t$ test, $p<0.01)$ and neighboring directions $(p<0.05)$. Further on, these aftereffects were completely washed out as the mean directional deviations across postlearning trials $6-10$ or even across later trials were no longer significant (supplemental Fig. 1D, right, available at www.jneurosci.org as supplemental material) $(t$ test, $p>0.10)$. To extract memory traces that persisted after learning, we thus evaluated movement parameters (i.e., hand trajectories, velocities, reaction times) and neuronal activity cor- 
Table 1. Sample proportion of changes in tuning properties of motor cortical cells

\begin{tabular}{|c|c|c|c|c|}
\hline & \multicolumn{2}{|l|}{ Preparatory } & \multicolumn{2}{|c|}{ Movement-related } \\
\hline & $\mathrm{FFv}$ & FFnv & $\mathrm{FFv}$ & FFnv \\
\hline \multicolumn{5}{|l|}{ Directionally tuned cells } \\
\hline Total & 150 & 128 & 243 & 237 \\
\hline Monkey A & 92 & 82 & 146 & 124 \\
\hline Monkey B & 58 & 46 & 97 & 113 \\
\hline \multicolumn{5}{|l|}{ FF-modulated cells } \\
\hline Total (late phase only) & 40 & 39 & 93 & 90 \\
\hline Monkey A & 21 & 14 & 50 & 26 \\
\hline Monkey B & 19 & 25 & 43 & 64 \\
\hline \multicolumn{5}{|l|}{ Mean firing rate } \\
\hline FF-modulated cells & $26 / 40$ & $19 / 39$ & $78 / 93$ & $78 / 90$ \\
\hline Non-FF-modulated cells & 107 & 106 & 143 & 153 \\
\hline \multicolumn{5}{|l|}{ Tuning changes } \\
\hline Total & $36 / 150(24 \%)$ & $26 / 128(20 \%)$ & 97/243 (40\%) & 96/237 (41\%) \\
\hline FF-modulated cells & 8/36 (22\%) & 6/26 (23\%) & 37/97 (38\%) & $40 / 96(42 \%)$ \\
\hline Non-FF-modulated cells & $28 / 36(78 \%)$ & $20 / 26(77 \%)$ & $60 / 97(62 \%)$ & $56 / 96(58 \%)$ \\
\hline \multicolumn{5}{|l|}{ PD shifts } \\
\hline Total & $26 / 36(54 \%)$ & $16 / 26(44 \%)$ & 60/97 (57\%) & $68 / 96(66 \%)$ \\
\hline FF-modulated cells & $3 / 26(12 \%)$ & $4 / 16(25 \%)$ & $25 / 60(42 \%)$ & $31 / 68(46 \%)$ \\
\hline Non-FF-modulated cells & $23 / 26(88 \%)$ & $12 / 16(75 \%)$ & $35 / 60(58 \%)$ & $37 / 68(54 \%)$ \\
\hline \multicolumn{5}{|l|}{ Amplitude } \\
\hline Total & $17(35 \%)$ & $15(42 \%)$ & $72(68 \%)$ & $59(57 \%)$ \\
\hline FF-modulated cells & $6 / 17(35 \%)$ & $2 / 15(13 \%)$ & $29 / 72(40 \%)$ & $24 / 59(41 \%)$ \\
\hline Non-FF-modulated cells & $11 / 17(65 \%)$ & $13 / 15(87 \%)$ & $43 / 72(60 \%)$ & $35 / 59(59 \%)$ \\
\hline
\end{tabular}

Dataset includes cells that were directionally tuned in both prelearning and postlearning blocks $(N=758)$. FFv, Force field with visual feedback; FFnv, force field without visual feedback.

responding to the postlearning trials after the washout of aftereffects attributable to learning (i.e., from the sixth trial onwards). For these trials, we found that movement parameters were comparable before and after learning ( $t$ test, $p>0.10$ ) (supplemental Fig. $1 A, B$, available at www.jneurosci.org as supplemental material). While behavior in null-field reaches remained unchanged after learning, movement-related activity of 156 of 183 (85\%) FF-modulated cells significantly changed from prelearning to postlearning (joint vision and nonvision, Mann-Whitney, $p<$ 0.05) (Table 1, Mean firing rate). Furthermore, the tuning properties of 77 of $183(42 \%)$ FF-modulated cells were significantly modified (permutation test, $p<0.05$ ) (Table 1 , Tuning changes).

\section{Rate modulation}

Neuronal activity during movement execution

Force field-modulated cells retain their adapted activity after learning. We then evaluated whether the rate modulation of FFmodulated cells from prelearning to postlearning were specific to the newly learned task. Figure 2, $A$ and $B$, shows perievent time histograms (PETHs) of two examples of FF-modulated cells. Each panel shows the prelearning and postlearning PETHs for each of the eight target directions. For the LD, PETHs corresponding to the early (first 20) and late FF trials (trials 61-80) are also shown (plots with orange arrows). The neuron in $A$ retained its decreased activity during adaptation (green and orange) through the postlearning block (blue). In Figure $2 B$, some of the increased activity of the neuron observed in late force field persisted through postlearning. In general, during null-field reaches to the LD, most cells with nPD opposite to the FF direction maintained the elevated firing rate acquired during adaptation (Fig. $2 C$, cyan-shaded area) (binomial test, $p=0.001$ ). Likewise, cells with $\mathrm{nPD}$ similar to the FF direction maintained the decreased rate that they exhibited during the adaptation block (Fig. 2C, magenta-shaded area) ( $p=0.001)$. Thus, the postlearning activity at the learned direction took off from the newly acquired activity pattern, suggesting that these changes may be associated with early memory of the learned task.
For directions other than the learned direction, the changes in the postlearning firing rates compared with prelearning are also shown by the PETHs of Figure 2. Note that the changes of postlearning activity in these directions showed consistent relation between the PD of the cells and the LD. They closely resembled the adaptive pattern (i.e., cosine-like modulations that we had shown during adaptation) (Arce et al., 2010). This adaptive pattern is depicted in the center of each panel $(A, B)$ of Figure 3 . The pattern of the postlearning modulations is shown in the eight radial plots in Figure 3. These plots show the postlearning population modulation index ( $y$-axis) as a function of the nPD estimated for the postlearning block ( $x$-axis, shown in ranges). The figure quantifies the postlearning modulation index, showing that it is maximal for counterfield cells ( $\mathrm{nPD}$ of -46 to $-135^{\circ}$ ) and minimal for with-field cells (nPD of 46 to $135^{\circ}$ ). The overall pattern of modulations of all cells well fit a cosine model (for the LD: $R^{2}=$ 0.97 and 0.80 for vision and nonvision, respectively, $p<0.01$; and for all other directions: $\left.R^{2} \geq 0.70, p<0.05\right)$. These findings suggest that FF-modulated cells retained the activity weights that were acquired during adaptation.

When learning was with VFB (Fig. 3A), seven of eight directions had a significant fit to cosine. Without VFB (Fig. $3 B$ ), the adaptive pattern was present in only a few of the nonlearned directions (four of eight). The more generalized pattern with VFB may reflect differences in the stabilization of motor memories based on the available sensory feedback. Indeed, Figure 4 shows that when the modulation indexes of the cells per $\mathrm{nPD}$ range were pooled across all target directions, the modulation indexes were significantly lower in the nonvision than in the vision condition (ANOVA, $p<0.0001$ ).

Changes in firing rates of non-force field-modulated cells. We also found that other directionally tuned cells also changed their firing rates from prelearning to postlearning even though they did not show rate modulation during force field adaptation. We refer to them as "non-FF-modulated cells." We determined whether such rate modulation in the postlearning were learning 

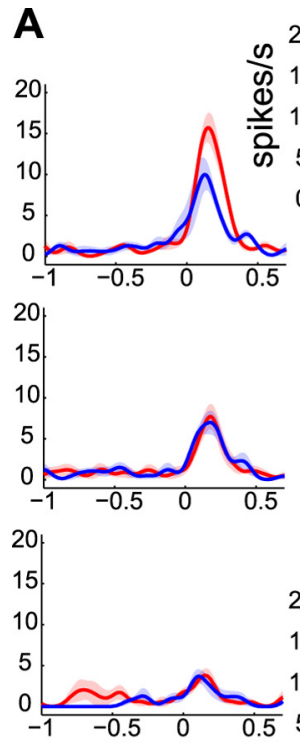
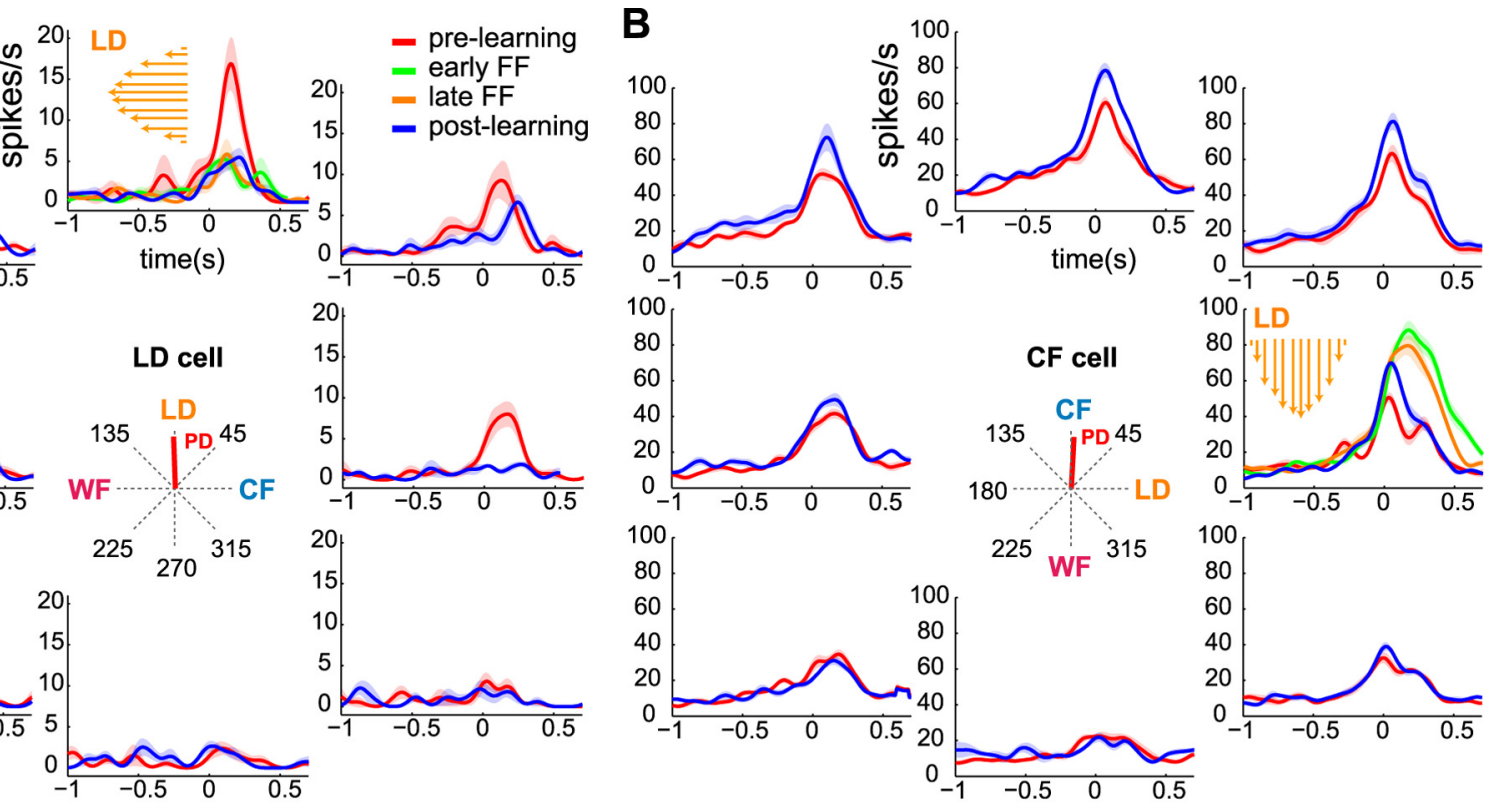

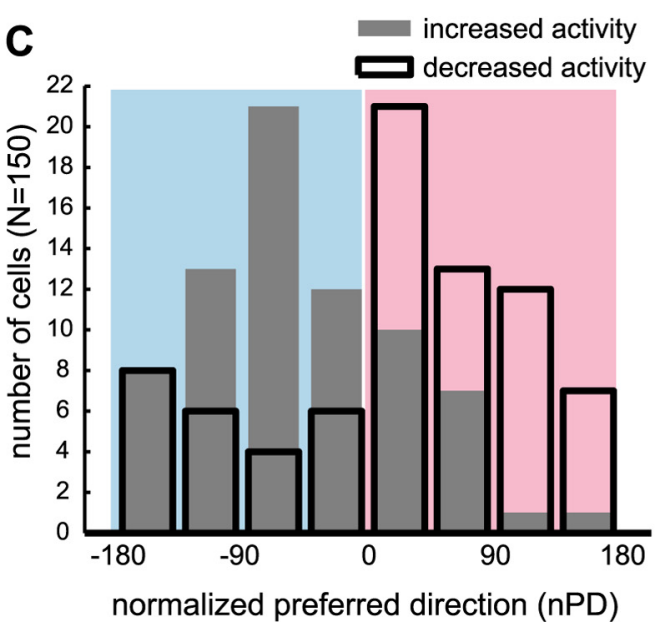

Figure 2. Changes in the activity of M1 neurons after adaptation to force field. $A, B$, PETHs (and $\pm 1 \mathrm{SE}$ ) smoothed by a $50 \mathrm{~ms}$ Gaussian kernel during prelearning (red) and postlearning (blue) reaches to eight directions and during early (green) and late (orange) force field reaches to targets at 90 and $0^{\circ}$ (subplots with orange arrows indicating FF direction). PETHs are aligned at movement onset ( $0 \mathrm{~s}$ ). Example of a "LD cell" whose actual PD (central plot, red line) is $2^{\circ}$ from the LD $(\boldsymbol{A})$ and a "CF cell" whose actual PD (red line) is $-87^{\circ}$ from the LD and opposite to the clockwise force field $(\boldsymbol{B})$. The PD of the neuron in $\boldsymbol{A}$ shifted $\left(15^{\circ}\right)$ from prelearning to postlearning, whereas the tuning amplitude of the neuron in $\boldsymbol{B}$ increased (permutation test, $\left.p<0.05\right)$. $\boldsymbol{C}$, Histogram shows the number of FF-modulated cells ( $y$-axis) that increased (gray-filled) or decreased (black) their postlearning activity according to the normalized preferred direction ( $x$-axis). The color-shaded areas denote cells with nPD opposite (cyan) or similar (magenta) to the FF direction. Data combine similar results in both feedback conditions.

specific. We found that the non-FF-modulated cells did not exhibit a consistent PD-dependent activity pattern similar to the adaptive pattern during null-field reaches toward the LD nor across any other direction. This was apparent in the nonsignificant cosine-fits on the modulation indexes ( $F$ test, $p>0.10$ ). Moreover, the mean modulation indexes were generally lower than were observed in the FF-modulated cells (Fig. 4, compare solid, dashed lines).

Changes in firing rates of cells in the control repetition sessions. To further verify that the postlearning changes of FF-modulated cells were learning related, we conducted control sessions $(n=$ 10 ) before the monkeys experienced any force field. In these sessions, the structure of trial blocks was similar to the learning sessions; however, instead of the learning block, monkeys performed repeated null-field reaching to one target with or without VFB. We analyzed a total of 166 directionally tuned cells (vision, 75; nonvision, 91). Differences in discharge rates across the three trial blocks were assessed using one-way ANOVA with post hoc Tukey-Kramer correction for multiple comparisons. During the repetition block, firing rates of 64 of 166 (39\%) cells were found to be different in the repeated (one) direction compared with reaches made to the same target during the prerepetition block (ANOVA, $p<0.05$ ). Unlike the adaptive modulation, rate modulation in the repetition block did not exhibit a similar linkage between the nPD and modulation indexes of the cells ( $F$ test, $p>$ $0.10)$. When comparing prerepetition to postrepetition, we found cells that also changed their firing rates significantly. However, their modulation indexes did not depend on the PD of the cells ( $F$ test, $p>0.10)$.

Neural activity during movement preparation

Modulation of the preparatory activity during FF adaptation was found in 109 of 278 (39\%) directionally tuned cells. The rate modulation in these cells also depended on the nPD of the cells ( $F$ 
A Vision
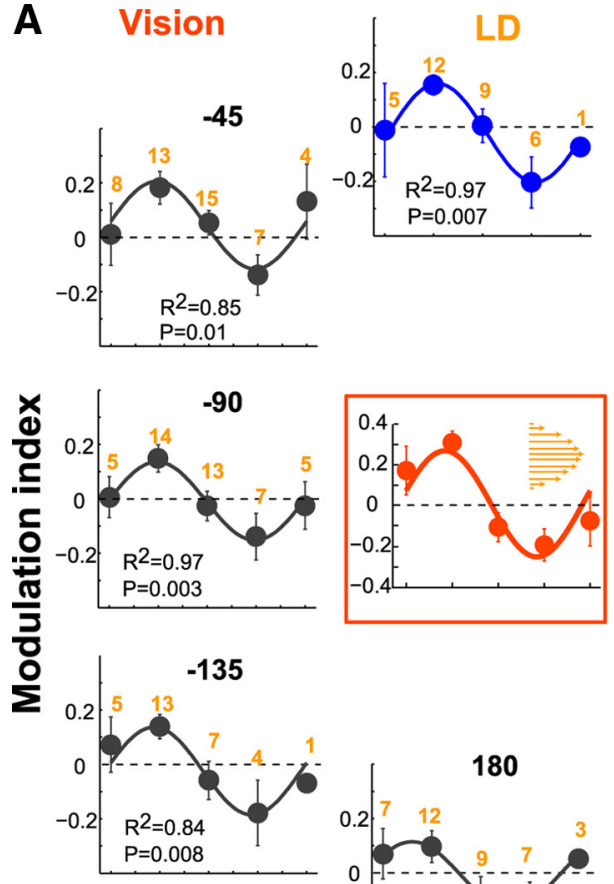

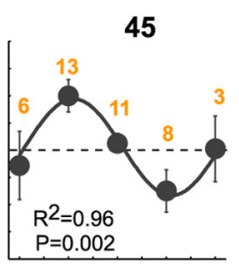

B
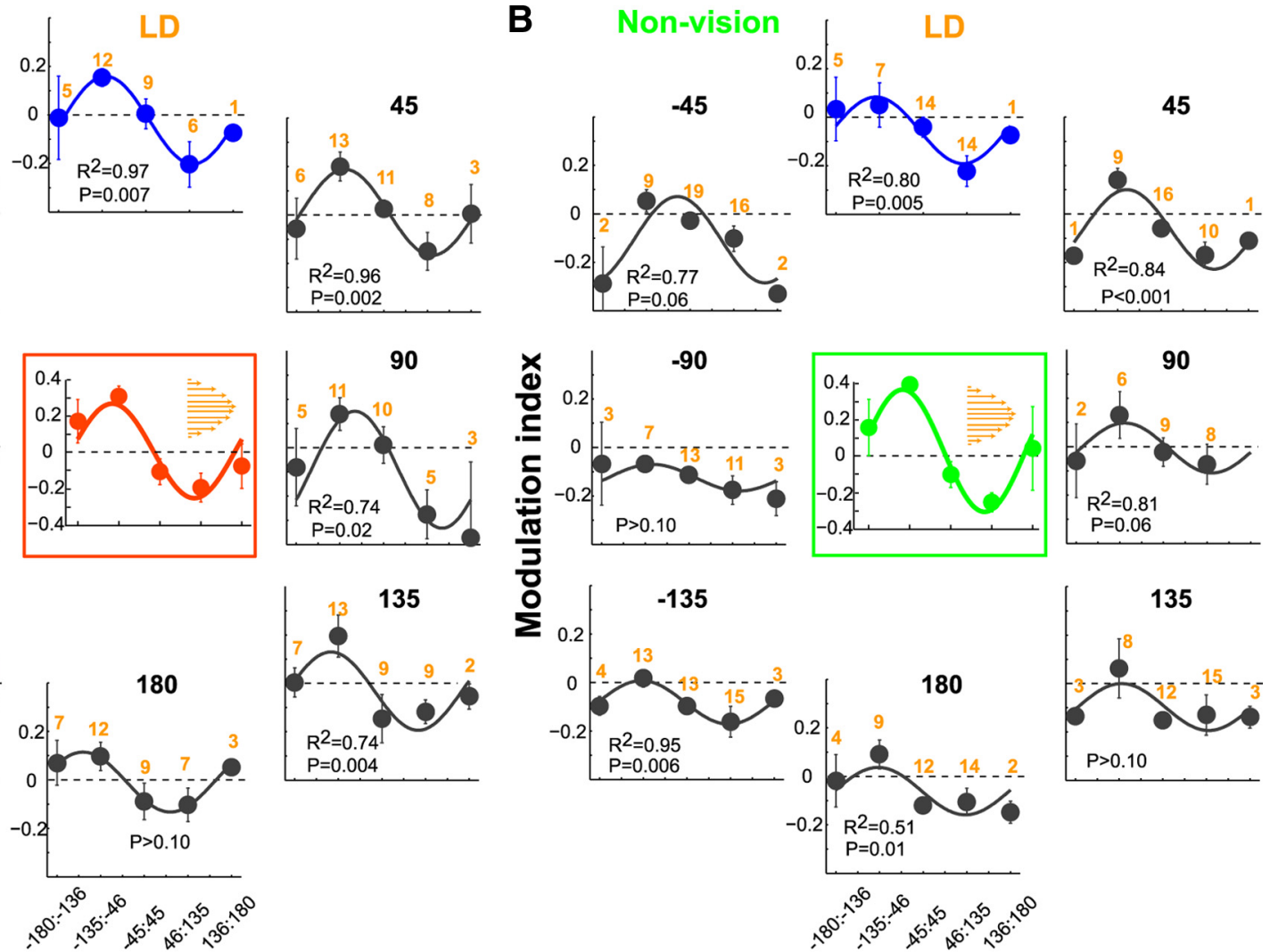

normalized PD $\left({ }^{\circ}\right)$

Figure 3. Retained coactivation of cell ensembles after adaptation to force fields with visual feedback $(\boldsymbol{A})$ and without $(\boldsymbol{B})$. The postlearning pattern is shown in the radial plots, and the adaptive pattern (recorded during FF adaptation) is shown in the central boxed plots. Note that the central plot and the radial plots are quite similar (see text for analysis). Radial plots: For each panel, the eight radial plots correspond to target directions organized according to the distance from the LD and the FF direction (i.e., positive directions correspond to directions similar to FF, whereas negative directions correspond to directions opposite to FF). This was done to pool the data across all recording sessions with varying LD and FF direction. Each radial plot relates the population modulation indexes ( $y$-axis, mean $\pm 1 \mathrm{SE}$ ) to the $\mathrm{nPD}$, which corresponds to the angular distance of the PD of the cells from the LD ( $x$-axis, shown in ranges). Cosine-fit on the population modulation index significant $R^{2}$, and corresponding $p$ level are shown (cosine-fit significance set at $p<0.05$ ). The orange numbers on top of the cosine-fit denote the number of cells in each nPD range. Data include cells that were modulated by force field in the late adaptation phase and had significant rate differences between prelearning and postlearning. Central plots: For each panel, the central plot corresponds to the learned target direction. It relates the population modulation index (i.e., rate changes between prelearning and adaptation blocks) to the nPD as shown in the radial plots.

A

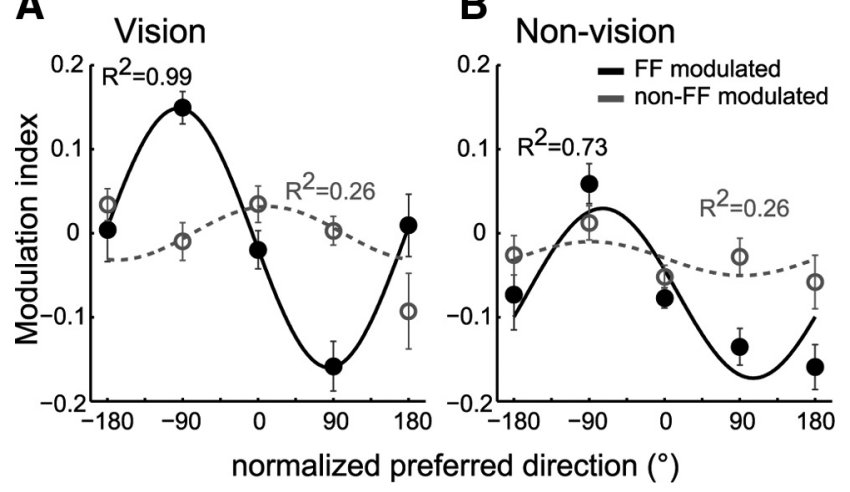

Figure 4. Postlearning pattern differs between force field-modulated and non-force fieldmodulated cells. Modulation indexes per nPD range were averaged across all target directions in vision $(\boldsymbol{A})$ and nonvision $(\boldsymbol{B})$ conditions and separately for FF-modulated (black) and non-FFmodulated cells (gray). Corresponding cosine-fits and $R^{2}$ are shown. Error bars denote $\pm 1 \mathrm{SE}$.

test, $p<0.001)$. However, the cosine profile was different from that observed for the movement-related activity; peak negative modulation corresponded to cells with nPD near the LD. After adaptation, 57\% (45 of 79) of FF-modulated cells showed significant rate modulation (Table 1, Mean firing rate). Because of the small sample size, we evaluated the dependence of the postlearning modulation indexes on the nPD of the cells without binning into ranges. For both feedback conditions, the relationship of modulation indexes with the nPD of the cells during this epoch showed a good fit to cosine and approximated the profile found in the movement-related activity $(F$ test, $p<0.01)$.

\section{Changes in tuning parameters}

Over $40 \%$ of the population of directionally tuned cells showed prelearning to postlearning changes in their tuning properties (Table 1, Tuning changes). These changes were either in the PD and/or tuning amplitude of the cell.

Reorganization of preferred directions: PD shifts from prelearning to postlearning

The preferred directions of all directionally tuned cells before and after learning were uniformly distributed in all cases (supplemental Fig. 2, available at www.jneurosci.org as supplemental material) (Rayleigh's test, $p>0.05$ ) except for the movement epoch of the prelearning block of the nonvision condition (Rayleigh's test, $p=0.03$ ). At the single-cell level, significant PD shifts were found (Table 1) (permutation test, $p<0.05$ ), comprising 15\% (42 of 278 ) of the entire neuronal population for movement preparation and 27\% (128 of 480) for movement execution (joint vision 
A
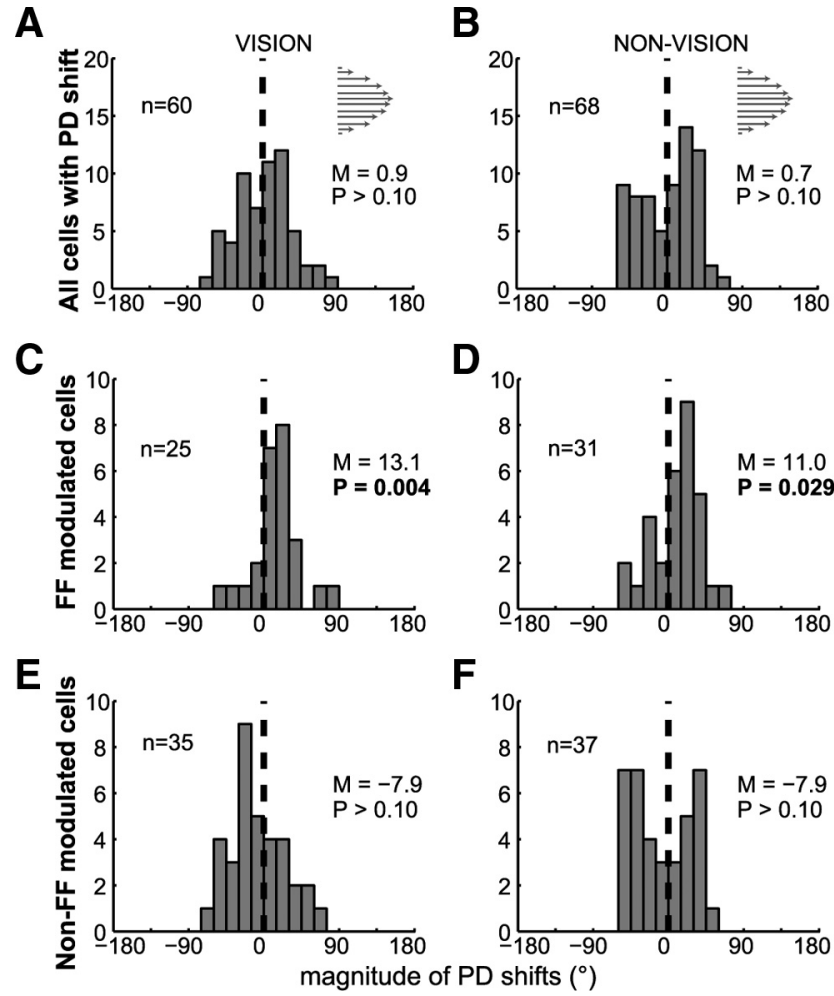

Figure 5. Preferred direction shifts in the population of force field-modulated cells. $\boldsymbol{A}, \boldsymbol{B}$, Distribution of all cells with significant PD shifts during the movement epoch of the vision and nonvision conditions. $\boldsymbol{C}-\boldsymbol{F}$, As in $\boldsymbol{A}$ and $\boldsymbol{B}$, shown separately for FF-modulated $(\boldsymbol{C}, \boldsymbol{D})$ and nonFF-modulated $(\boldsymbol{E}, \boldsymbol{F})$ cells. Mean PD shifts $(\mathrm{M})$ and $p$ levels are also shown. $n$ denotes the number of cells in each condition; the arrows indicate FF direction.

and nonvision). For both epochs, the mean PD shifts of these subpopulations were not significantly different from zero (Fig. $5 A, B)(t$ test, $p>0.10)$. These results are consistent with previous reports (Li et al., 2001; Rokni et al., 2007) and may explain the similar kinematics of reaching movements before and after learning.

We then examined the population of FF-modulated cells that showed significant PD shifts. For the population of FFmodulated cells, the mean PD shifts were not significant during the preparatory epoch (sign test, $p>0.10$ ) but were significant in the movement epoch of both feedback conditions (Fig. $5 C, D$ ) ( ign test, vision, $p=0.004$; nonvision, $p=0.02$ ). The mean PD rotated in the force field direction, with most cells rotating their PDs in the direction of the force field (Fig. 6A-D) (binomial test, $p<0.001)$. The direction and magnitude of PD shifts were similar across feedback conditions (Mann-Whitney, $p>0.10$ ). Note that the direction of PD shifts from prelearning to postlearning was similar to that described for PD shifts from prelearning to learning ( $\mathrm{Li}$ et al., 2001). This suggests that the FF-modulated cells may have retained the new PD acquired during learning, equivalent to the memory-I cell described in the cited study. Alternatively, as we noted previously for the adaptive changes (Arce et al., 2010), the PD shifts may not necessarily reflect changes in the directional tuning properties of the cells but a change in the "learned" intended movement direction. Either way, both possibilities reflect learning-related changes expressed by cells that showed rate modulation during adaptation.

Figure 6, $E$ and $F$, illustrates the proportion of FF-modulated cells that significantly rotated their PDs. Unexpectedly, PD rotations were observed across cells from different PDs and not only in some cells with a particular PD. Without VFB, PD shifts were more frequent (binomial, $p<0.05$ ) with LD cells (13 of 31) than CF cells ( 7 of 31), whereas with VFB, the proportion of CF cells (7 of 25$)$ and LD cells ( 8 of 25) were similar (binomial, $p>0.10$ ). The differences in the population profile between feedback conditions mirror those observed during adaptation (Arce et al., 2010).

For the population of non-FF-modulated cells, the mean PD shifts were not significant (Fig. $5 E, F)(p>0.10)$. In contrast to FF-modulated cells, the PD shifts of non-FF-modulated cells were equally distributed between PD shifts in the same or opposite direction to the force field. These findings suggest that the PD shifts do not reflect changes that relate to learning. To further evaluate whether the PD shifts in FF-modulated cells were associated with learning, similar analyses were performed on the control repetition sessions. Some of these cells also showed PD shifts from the prerepetition to the postrepetition block. However, in contrast to the learning experiment, PD shifts for these cells were not significant (sign test, $p>0.10$ ). Together, the PD shifts in the non-FF-modulated cells and the cells in the control repetition reflect ongoing fluctuations of single-cell properties, consistent with previous observations (Rokni et al., 2007). Thus, both findings provide evidence that the features of the changes in FFmodulated cells indicate a learning-specific effect on top of the "background changes" and early motor memory encoding.

\section{Postlearning changes in tuning amplitude}

Over $20 \%$ of the entire neuronal population exhibited a significant change in tuning amplitude from prelearning to postlearning (Fig. 7) (permutation, $p<0.05$ ). To evaluate postlearning changes in amplitude, we calculated a normalized index. As a population, no significant changes were found in the preparatory epoch for either feedback condition (sign test, $p>0.10$ ). For the movement epoch, only the mean tuning amplitude of the population of cells in the no-vision condition significantly decreased (Fig. $8 A$ ) (sign test, $p=0.04$ ). The decrease in amplitude was not accompanied by significant changes in the $R^{2}$ or the offset of these cells after learning (Mann-Whitney, $p>0.10$ ). When only the population of FF-modulated cells were tested, no net change in tuning amplitude was observed in either feedback condition (sign test, $p>0.10$ ).

Unlike the PD shifts that were predominantly in the direction of the force field, the changes in tuning amplitudes showed different trends across feedback and nPD ranges (Fig. $8 \mathrm{~A}$ ) (ANOVA interaction between feedback $\left.{ }^{\star} \mathrm{nPD}, p=0.009\right)$. We then compared the effects of feedback and nPD ranges on the tuning amplitudes of FF-modulated cells during the movement epoch. During the prelearning block, the tuning amplitudes were similar across feedback conditions (Mann-Whitney, $p>0.10$ ). After learning, we found that the effect of feedback on the changes in tuning amplitude was different at different $\mathrm{nPD}$ ranges (Fig. $8 \mathrm{~B}$ ) (ANOVA interaction between feedback ${ }^{*} \mathrm{nPD}, p=0.03$ ). Comparisons made for each of the $\mathrm{nPD}$ ranges revealed opposite effects of feedback for CF and WF cells; whereas the amplitude of CF cells increased in vision, it decreased in nonvision (MannWhitney, $p=0.01$ ) (Fig. 7, compare $A, D$ ). The reverse was true for the WF cells $(p=0.02)$ (Fig. 7, compare $C, F)$.

With regards to the population of non-FF-modulated cells, the effects of feedback and $\mathrm{nPD}$ ranges were not significant (Fig. 8C) (ANOVA, $p>0.05$ ), nor was the interaction between them (ANOVA, $p>0.10$ ). A similar trend of opposite effects in CF cells of vision and nonvision conditions was, however, apparent. 


\section{Discussion}

Summary of findings

This study compared the directional tuning properties of motor-cortical neurons before and after adaptation to force field. Our experiments were designed to extend a similar set of studies by Bizzi and colleagues (Gandolfo et al., 2000; Li et al., 2001). Here, we used local adaptation wherein monkeys experienced force field in one direction only during adaptation. We found that cell ensembles, which were modulated during adaptation [i.e., FF-modulated cells from the study by Arce et al. (2010)], maintained their newly acquired PD-dependent rate modulation (peak positive modulation for cells with PD opposite to the force field direction and peak negative modulation for cells with $\mathrm{PD}$ along the force field direction). The second finding is that the PD of this subpopulation were shifted in the direction of the force field after learning [like the memory I cells reported by Li et al. (2001)]. Last, we found changes in tuning amplitudes that differed across feedback conditions, consistent with the different learned trajectory shapes.

\section{Representation of motor memory by cell ensembles}

Previous studies have shown persistent neuronal changes after adaptation to novel sensorimotor transformations. These changes have been described as improved directional information (Paz and Vaadia, 2004), maintenance of newly acquired color sensitivity (Zach et al., 2008), and PD shifts at the single-cell level (Li et al., 2001). Here, we show a novel finding of sustained combined activity of cell ensembles that takes off from a recent learning episode. As such, it may suggest the formation of a functional cell assembly during learning or a modified interaction among members of an already existing functional assembly (Vaadia et al., 1995; Pastalkova et al., 2008) (for review, see Harris, 2005). This hypothesis suggests that the new or modified functional cell assembly is structured to generate the desired population directional signal for the compensatory force (Arce et al., 2010). The increased neuronal excitability and the new firing association would render these cells more easily activated and the connections among them stronger. Thus, it is plausible that neuron members would then continue to be active together when performing reaching movements in later exposures to force field and even in the absence of a force field. Various mechanisms were suggested that support this type of adaptation including the corticostriatal system. Evidence from different types of experiments suggests that it can function as an adaptivelearning system (for review, see Wickens, 2009).
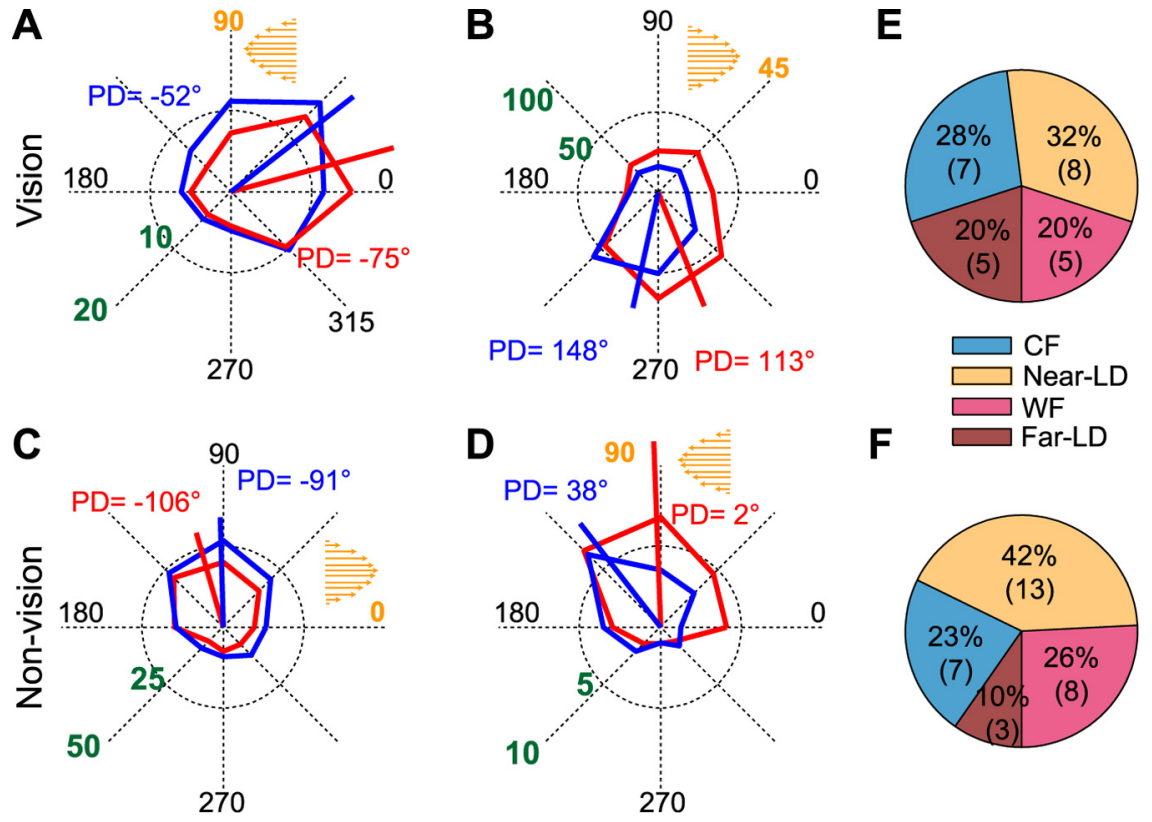

Figure 6. Reorganization of preferred directions during the movement epoch. $A-D$, Polar plots of mean firing rates of single units that significantly shifted their PDs from prelearning (red line) to postlearning (blue line). Normalized PD corresponding to the prelearning (red number) and postlearning (blue number) blocks are shown. The orange arrows indicate learned FF direction. Green numbers on the radius denote firing rates (spikes/second). $\boldsymbol{E}, \boldsymbol{F}$, Proportion of FF-modulated cells per $\mathrm{nPD}$ range that showed significant PD shifts.
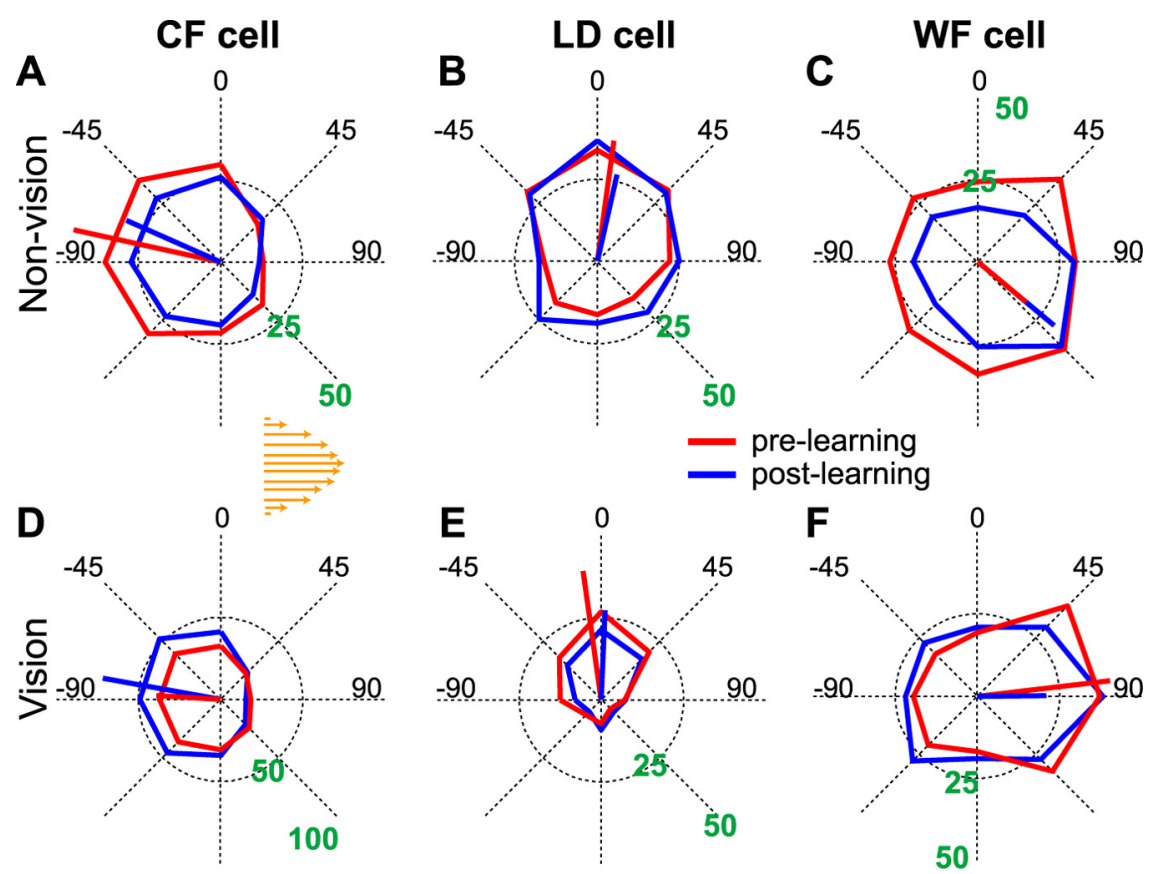

Figure 7. Tuning amplitude of M1 neurons changes after learning. Polar plots of movement-related activity of FF-modulated cells during prelearning (red) and postlearning (blue). Cell examples from different nPD ranges of nonvision $(\boldsymbol{A}-\boldsymbol{C})$ and vision $(\boldsymbol{D}-\boldsymbol{F})$ conditions. The prelearning and postlearning PDs (red and blue lines, respectively) correspond to the PD distance from the $\mathrm{LD}\left(0^{\circ}\right)$ and for a clockwise force field (orange arrows). PD shift was also found significant in $\boldsymbol{A}$ and $\boldsymbol{E}$, but not in $\boldsymbol{B}-\boldsymbol{D}$ and $\boldsymbol{F}$. The green numbers on the radius denote firing rates (spikes/second).

The recently acquired activity pattern was sustained during the performance of a well known task of center-out reaches that lasted for $\sim 12-15$ min after adaptation. Thus, the sustained coactivation of cell ensembles may also underlie a novel representation of early memory by cell ensembles in the motor cortex. The following findings support this claim. First, the rate modulation of these cell ensembles in postlearning closely matched the adap- 

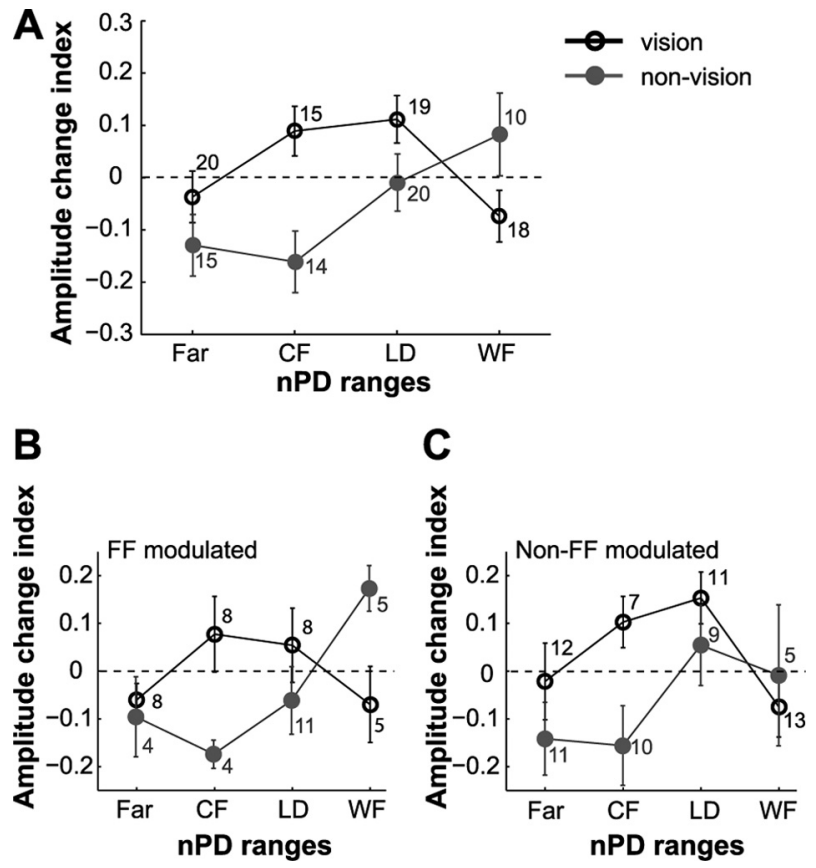

Figure 8. Postlearning changes in tuning amplitude differ across nPD ranges and feedback. $\boldsymbol{A}$, Mean amplitude change indexes as a function of the $\mathrm{nPD}$ ranges. Shown for the movement epoch of the population of cells that showed significant amplitude change for vision (black) and nonvision (gray). $\boldsymbol{B}, \boldsymbol{C}$, Data in $\boldsymbol{A}$ separated into FF-modulated and non-FF-modulated cells, respectively. The number of cells in each $\mathrm{nPD}$ range is shown. The vertical bars denote $\pm 1 \mathrm{SE}$.

tive pattern, indicating the persistence of the adaptive pattern that was not in response to movement errors. Second, this pattern of activity persisted at the learned direction and occurred at directions other than the learned one while performing null-field reaches. This reflects a generalized pattern of activation, thus going beyond mere persistence of the local learning. Last, when the monkeys were exposed again to force field ("retest"), the cells showed similar response properties as in the adapted phase of the first exposure. This suggests successful retrieval of an early motor memory trace, consistent with the observed behavioral and neuronal savings during retest.

Learning studies in the hippocampus have shown that cells with similar place fields that were coactive during wake continue to fire together during sleep (Harris et al., 2003; O'Neill et al., 2008). Such reactivation of memory traces by cell ensembles during inactive states may not be limited within a brain area but may span large areas of the primate neocortex (Hoffman and McNaughton, 2002). Thus, similar mechanisms may subserve the retention of early memories in the motor cortex. Indeed, rapid memory encoding and consolidation was suggested to occur in the neocortex (Tse et al., 2007; Wang and Morris, 2010) as opposed to previous views that memories are only transferred to the neocortex for later consolidation. Future studies could also examine whether early representation of motor memories by cell ensembles later evolve into a stable and consolidated form in the motor cortex. The finding of a stable motor cortical map across many days of neuroprosthetic control supports its feasibility (Ganguly and Carmena, 2009).

On the role of sensory feedback in adaptation and memory Firing patterns were modulated in a similar manner when adapting to force field either with or without visual trajectory feedback. The sustained pattern of activity after learning was also similar in the two feedback conditions. This provides support for the notion that sensory stimuli do not determine the firing patterns but rather modulate them (Llinás and Paré, 1991). In our experiments, this modulation took the form of differential profiles of the population of cells recruited during adaptation; with VFB, counterfield cells were predominant, whereas without VFB, cells with PD near the LD topped the distribution (Arce et al., 2010). Once again, for postlearning changes, the contribution of sensory feedback was expressed in different cell groups that showed increases in tuning amplitudes. With VFB, tuning amplitude increased in CF cells, whereas without VFB, it increased in WF cells. The different responses between these two cell groups parallel the suggestion of a reweighting strategy (Jarosiewicz et al., 2008), which, in the case here, applies to "preferred" cell groups depending on the feedback context in which learning took place. Increasing the tuning amplitude increases the contribution of the cell to the population vector. Thus, the increased tuning amplitude of CF cells may reflect the adaptive strategy that yields straight trajectories in force field with VFB, whereas that of WF cells may reflect the adaptive strategy that yields the curved trajectories learned without VFB. Together, the changes in FF-modulated cells averaged out, thereby producing no net effect on the motor output during null-field reaches, which were typically straight in both feedback conditions. This indicates preservation of the appropriate motor performance in null-field reaches while learning-specific changes persist in some cells to constitute motor memories of other learned tasks. Overall, the results reflect the different strategies adopted as a function of the available sensory feedback, consistent with our previous findings (Arce et al., 2009, 2010).

\section{Learning-specific versus random changes}

We have also shown prelearning to postlearning changes in the tuning properties of the population of cells that were not modulated during force field adaptation (i.e., non-FF-modulated cells). We have suggested that the changes in the non-FFmodulated cells reflect "random" background changes as opposed to the learning-specific changes in the tuning properties of FF-modulated cells. The learning-specific effects that remain after the learning episode were thus "unmasked" when the population was divided into FF-modulated and non-FF-modulated cells. In previous studies, the entire population of cells was assessed when comparing changes from prelearning to postlearning, thereby plausibly masking the learning-specific changes ( $\mathrm{Li}$ et al., 2001; Rokni et al., 2007). It was thus suggested that the prelearning to postlearning changes formed part of irrelevant changes attributable to plasticity noise (Rokni et al., 2007). Here, we teased out the prelearning to postlearning changes in the tuning properties of the cells into learning-specific and background changes. We propose here that the learning-specific changes during postlearning underlie retention of the representation of the newly learned dynamics. Functionally, the variability in the tuning properties of the non-FF-modulated cells may afford the plasticity needed for fast adaptation while ensuring that the learning-specific changes do not interfere with the performance of already known tasks. Furthermore, those non-FFmodulated cells that showed tuning changes that were similar to FF-modulated cells may be potentiated for easy recruitment during future exposures to force fields or related tasks.

\section{Conclusion}

In sum, we have shown sustained learning-specific changes in the response properties of cell ensembles in the primate motor cor- 
tex. Our findings suggest a novel representation of motor memory by the same cell ensembles that were activated during adaptation, indicating that similar neural circuits in the motor cortex underlie learning and the generation of early motor memories.

\section{References}

Arce F, Novick I, Shahar M, Link Y, Ghez C, Vaadia E (2009) Differences in context and feedback result in different trajectories and adaptation strategies in reaching. PLoS ONE 4:e4214.

Arce F, Novick I, Mandelblat-Cerf Y, Israel Z, Ghez C, Vaadia E (2010) Combined adaptiveness of specific motor cortical ensembles underlies learning. J Neurosci 30:5415-5425.

Attwell PJ, Cooke SF, Yeo CH (2002) Cerebellar function in consolidation of a motor memory. Neuron 34:1011-1020.

Baraduc P, Lang N, Rothwell JC, Wolpert DM (2004) Consolidation of dynamic motor learning is not disrupted by rTMS of primary motor cortex. Curr Biol 14:252-256.

Dave AS, Margoliash D (2000) Song replay during sleep and computational rules for sensorimotor vocal learning. Science 290:812-816.

Gandolfo F, Li C, Benda BJ, Schioppa CP, Bizzi E (2000) Cortical correlates of learning in monkeys adapting to a new dynamical environment. Proc Natl Acad Sci U S A 97:2259-2263.

Ganguly K, Carmena JM (2009) Emergence of a stable cortical map for neuroprosthetic control. PLoS Biol 7:e1000153.

Han JH, Kushner SA, Yiu AP, Hsiang HL, Buch T, Waisman A, Bontempi B, Neve RL, Frankland PW, Josselyn SA (2009) Selective erasure of a fear memory. Science 323:1492-1496.

Harris KD (2005) Neural signatures of cell assembly organization. Nat Rev Neurosci 6:399-407.

Harris KD, Csicsvari J, Hirase H, Dragoi G, Buzsáki G (2003) Organization of cell assemblies in the hippocampus. Nature 424:552-556.

Hoffman KL, McNaughton BL (2002) Coordinated reactivation of distributed memory traces in primate neocortex. Science 297:2070-2073.

Jarosiewicz B, Chase SM, Fraser GW, Velliste M, Kass RE, Schwartz AB (2008) Functional network reorganization during learning in a brain-computer interface paradigm. Proc Natl Acad Sci U S A 105:19486-19491.

Ji D, Wilson MA (2007) Coordinated memory replay in the visual cortex and hippocampus during sleep. Nat Neurosci 10:100-107.

Karlsson MP, Frank LM (2009) Awake replay of remote experiences in the hippocampus. Nat Neurosci 12:913-918.

Karni A, Meyer G, Jezzard P, Adams MM, Turner R, Ungerleider LG (1995) Functional MRI evidence for adult motor cortex plasticity during motor skill learning. Nature 377:155-158.

Kassardjian CD, Tan YF, Chung JY, Heskin R, Peterson MJ, Broussard DM (2005) The site of a motor memory shifts with consolidation. J Neurosci 25:7979-7985.

Lebedev MA, Carmena JM, O’Doherty JE, Zacksenhouse M, Henriquez CS, Principe JC, Nicolelis MA (2005) Cortical ensemble adaptation to represent velocity of an artificial actuator controlled by a brain-machine interface. J Neurosci 25:4681-4693.

Li CS, Padoa-Schioppa C, Bizzi E (2001) Neuronal correlates of motor performance and motor learning in the primary motor cortex of monkeys adapting to an external force field. Neuron 30:593-607.

Llinás RR, Paré D (1991) Of dreaming and wakefulness. Neuroscience 44: 521-535.

Misanin JR, Miller RR, Lewis DJ (1968) Retrograde amnesia produced by electroconvulsive shock after reactivation of a consolidated memory trace. Science 160:554-555.

Montgomery SM, Buzsáki G (2007) Gamma oscillations dynamically couple hippocampal CA3 and CA1 regions during memory task performance. Proc Natl Acad Sci U S A 104:14495-14500.

Muellbacher W, Ziemann U, Wissel J, Dang N, Kofler M, Facchini S, Boroojerdi B, Poewe W, Hallett M (2002) Early consolidation in human primary motor cortex. Nature 415:640-644.

Nader K (2003) Memory traces unbound. Trends Neurosci 26:65-72.

Nader K, Hardt O (2009) A single standard for memory: the case for reconsolidation. Nat Rev Neurosci 10:224-234.

O’Neill J, Senior TJ, Allen K, Huxter JR, Csicsvari J (2008) Reactivation of experience-dependent cell assembly patterns in the hippocampus. Nat Neurosci 11:209-215.

Pastalkova E, Itskov V, Amarasingham A, Buzsáki G (2008) Internally generated cell assembly sequences in the rat hippocampus. Science 321: 1322-1327.

Paz R, Vaadia E (2004) Learning-induced improvement in encoding and decoding of specific movement directions by neurons in the primary motor cortex. PLoS Biol 2:E45.

Paz R, Boraud T, Natan C, Bergman H, Vaadia E (2003) Preparatory activity in motor cortex reflects learning of local visuomotor skills. Nat Neurosci 6:882-890.

Reijmers LG, Perkins BL, Matsuo N, Mayford M (2007) Localization of a stable neural correlate of associative memory. Science 317:1230-1233.

Ribeiro S, Nicolelis MA (2004) Reverberation, storage, and postsynaptic propagation of memories during sleep. Learn Mem 11:686-696.

Richardson AG, Overduin SA, Valero-Cabré A, Padoa-Schioppa C, PascualLeone A, Bizzi E, Press DZ (2006) Disruption of primary motor cortex before learning impairs memory of movement dynamics. J Neurosci 26:12466-12470.

Robertson EM (2009) From creation to consolidation: a novel framework for memory processing. PLoS Biol 7:e1000019.

Rokni U, Richardson AG, Bizzi E, Seung HS (2007) Motor learning with unstable neural representations. Neuron 54:653-666.

Silva AJ, Zhou Y, Rogerson T, Shobe J, Balaji J (2009) Molecular and cellular approaches to memory allocation in neural circuits. Science 326:391-395.

Stark E, Abeles M (2005) Applying resampling methods to neurophysiological data. J Neurosci Methods 145:133-144.

Stickgold R, Walker MP (2005) Memory consolidation and reconsolidation: what is the role of sleep? Trends Neurosci 28:408-415.

Thompson RF (2005) In search of memory traces. Annu Rev Psychol 56:1-23.

Tse D, Langston RF, Kakeyama M, Bethus I, Spooner PA, Wood ER, Witter MP, Morris RG (2007) Schemas and memory consolidation. Science 316:76-82.

Vaadia E, Haalman I, Abeles M, Bergman H, Prut Y, Slovin H, Aertsen A (1995) Dynamics of neuronal interactions in monkey cortex in relation to behavioural events. Nature 373:515-518.

Wang SH, Morris RG (2010) Hippocampal-neocortical interactions in memory formation, consolidation, and reconsolidation. Annu Rev Psychol 61:49-79, C1-4.

Wickens JR (2009) Synaptic plasticity in the basal ganglia. Behav Brain Res 199:119-128.

Wise SP, Moody SL, Blomstrom KJ, Mitz AR (1998) Changes in motor cortical activity during visuomotor adaptation. Exp Brain Res 121:285-299.

Zach N, Inbar D, Grinvald Y, Bergman H, Vaadia E (2008) Emergence of novel representations in primary motor cortex and premotor neurons during associative learning. J Neurosci 28:9545-9556. 